\title{
MODELO EXPERIMENTAL PARA MEDIR LA PRESIÓN INTRAOCULAR (PIO) DURANTE LASIK
}

\section{AN EXPERIMENTAL MODEL TO MEASURE REAL INTRAOCULAR PRESSURE (IOP) DURING LASIK}

\author{
HERNÁNDEZ-VERDEJO JL , BOLÍVAR-DE-MIGUEL G², ROMÁN-GUINDO JM³ \\ TEUS-GUEZALA MA ${ }^{4}$
}

\section{RESUMEN}

Objetivo: Diseñar un modelo para medir la presión intraocular real (PIO) durante el procedimiento LASIK (Laser Assisted in situ Keratomileusis) en ojos de cerdo.

Métodos: En este experimento se utilizó un transductor de presión sanguínea. Cada ojo se colocó sobre una superficie con soporte como para realizar el procedimiento quirúrgico. Los ojos se insuflaron con una solución glucosada al 5\% introducida a través del nervio óptico con el fin de conseguir una presión intraocular de inicio de entre $8-20 \mathrm{mmHg}$. En ese momento, un catéter de $27 \mathrm{G}$ se insertó desde la pars-plana hacia la cámara anterior. La PIO real se transmitió vía la columna líquida del catéter hacia un sensor externo. Después se colocó el anillo de succión y se realizó el corte. Durante todo el proceso, la PIO fue registrada mediante un amplificador ML 110 Bridge conectado al baro-transductor.

Resultados: Se midieron los valores de PIO real, en siete ojos de cerdo enucleados, durante el procedimiento LASIK. El modelo fue capaz de registrar instantáneamente los cambios de PIO inducidos por

\begin{abstract}
Purpose: To design a model to measure real IOP during lasik in pig eyes.

Methods: A reusable blood pressure transducer was used. Each eye was placed on a stand with sufficient support for the surgical procedure. The globes were inflated with $5 \%$ glucosated solution through the optic nerve to attain an IOP of 10-20 $\mathrm{mmHg}$. A 27-gauge catheter was then inserted from the pars plana to the anterior chamber cavity. Real IOP was transmitted via the catheter liquid column to an external sensor. A suction ring was applied and a flap was created. During the procedure, IOP was registered with the ML110 Bridge Amplifier connected to a baro transducer.
\end{abstract}

Results: Values of real IOP were measured in freshly enucleated pig eyes during the lasik procedure. The model was able to instantaneously register the changes in IOP induced by the application of a suction ring and the creation of a flap in the pig eyes with good reproducibility. At the end of the procedure the IOP values remained stable.

Conclusions: We have developed an experimental model to observe the course of suctioning and chan-

\footnotetext{
Recibido: 31/5/06. Aceptado: 25/7/07.

VISSUM Corporación Oftalmológica. Madrid. España.

1 Diplomado en Óptica y Optometría. VISSUM Madrid. EU de Óptica. Universidad Complutense de Madrid. Madrid. España.

2 Licenciada en Medicina. Hospital Universitario Príncipe de Asturias. Alcalá de Henares. Madrid. España.

3 Licenciado en Medicina. VISSUM Madrid.

4 Doctor en Medicina. VISSUM Madrid. Hospital Universitario Príncipe de Asturias. Alcalá de Henares. Madrid. España. Comunicación presentada parcialmente en el LXXXII Congreso de la S.E.O. (A Coruña 2006).
}

Correspondencia:

José Luis Hernández Verdejo

VISSUM Corporación Oftalmológica

C/. Santa Hortensia, 58

28002 Madrid

España

E-mail: jlhernan@opt.ucm.es 
la aplicación del anillo de succión y la creación del flap corneal con buena reproductibilidad. Al final del procedimiento, los valores de PIO resultantes permanecían estables.

Conclusiones: Hemos desarrollado un modelo experimental para observar simultáneamente durante la succión del ojo y la creación del flap los cambios de presión intraocular que se producen. Este modelo parece ofrecer con precisión los valores de PIO en la cámara anterior durante el procedimiento LASIK.

Palabras clave: PIO real, lasik, manometría, ojo cerdo, cámara anterior. ges in IOP simultaneously. This model seems to be accurate in obtaining IOP values from the anterior chamber cavity during lasik (Arch Soc Esp Oftalmol 2007; 82: 541-546).

Key words: Real IOP, Lasik, manometry, porcine eye, anterior chamber.

\section{INTRODUCCIÓN}

Disponer de un modelo con el que se pueda medir la PIO (presión intraocular) de forma sensible e inmediata cuando se realiza cirugía ocular es algo interesante al permitir el registro de los cambios de presión inducidos por las maniobras quirúrgicas. Las razones por las que estos experimentos no pueden llevarse a cabo en humanos tienen su justificación desde el punto de vista ético lo que anima al desarrollo de modelos animales que presenten suficientes similitudes anatómicas con el ojo humano.

Algunos autores han medido la PIO durante la cirugía refractiva corneal. Bissen-Miyajima $\mathrm{H}$ et al. (1) desarrollaron un modelo experimental para observar el curso de la succión y los cambios de PIO simultáneamente. Kasetsuwan $\mathrm{N}$ et al (2) midieron la PIO real durante la queratomileusis y determinaron la variabilidad del espesor de flap utilizando diferentes ajustes de presiones de succión. Sachs HG et al. (3) evaluaron la PIO durante el procedimiento LASIK en ojos de cerdo utilizando dos sistemas de microqueratomo diferentes.

En estos trabajos el sistema de medida que se utilizó, con algunas variaciones entre ellos, fue mediante la canulación de la cámara vítrea conectando ésta a un sensor de presión. Los valores de presión, por tanto, se registraron desde una zona del ojo no involucrada directamente en la maniobra quirúrgica.

Este hecho puede suscitar ciertas dudas en cuanto a la fiabilidad de las medidas dado que el vítreo mantiene una consistencia viscosa dificultándose de esta forma su movilidad a través de la columna de líquido empleada en estos sistemas de medida.
Por este motivo y con el fin de subsanar los lógicos inconvenientes que pueden tener los modelos previamente mencionados decidimos diseñar un modelo en el que los valores de PIO se registraran directamente en la cámara anterior del ojo, espacio ocupado por el humor acuoso, líquido de menor consistencia que el vítreo y más fácilmente movible.

\section{SUJETOS, MATERIAL Y MÉTODO}

En este modelo experimental se utilizaron ojos de cerdos enucleados y se evaluaron de manera prospectiva los cambios de PIO originados desde la aplicación del anillo de succión hasta el momento en que finaliza la creación del flap realizado éste mediante un láser de femtosegundo (IntraLase Corp. Irvine, CA).

Se utilizaron siete ojos de cerdo anatómicamente íntegros y libres de daño corneal tras haber sido verificado esto último mediante la lámpara de hendidura.

Los ojos fueron insuflados con una solución glucosada al 5\% a través del nervio óptico (de la misma manera que en el experimento de Kasetsuwan y col.) con el fin de obtener una PIO entre 15 y 20 $\mathrm{mmHg}$, confirmada mediante tonometría de aplanación Perkins (Clement Clarke, Essex, England), y colocados sobre una superficie con suficiente soporte como para poder realizar el procedimiento quirúrgico.

Se midió la PIO presente en la cámara anterior utilizando un catéter de 27G (Set ref: 387412 BD Valu-Set ${ }^{\mathrm{TM}}$ insertado intra-escleralmente de tal forma que no impidiera la aplicación del anillo de succión, y preservándose de que el extremo del catéter 
no tocara ninguna estructura intraocular durante todo el procedimiento quirúrgico.

Se obtuvieron las medidas de presión mediante un transductor desechable de presión sanguínea (MLT0380 Reusable BP Transducer, Power Lab, AD Instruments, Racine, WI). El transductor es un sensor externo de presión vascular conectado (en nuestro experimento la PIO en la cámara anterior) vía catéter-columna de líquido presente dentro de un tubo de silicona conectado al transductor. El transductor fue ajustado según las instrucciones del fabricante con el fin de asegurar una fijación precisa, un registro preciso de la PIO y la eliminación de toda posibilidad de presencia de aire dentro del sistema.

El transductor a su vez estaba conectado a un amplificador (ML110 Bridge Amplifier, AD Instruments) que mostraba instantáneamente la presión intraocular presente.

En esos momentos se aplicó el anillo de succión y se procedió a la creación del flap corneal. Todas las cirugía fueron llevadas a cabo en el mismo día por el mismo cirujano experimentado (JMR). Durante el procedimiento se registró de forma contínua la PIO, justo desde el momento de aplicación del anillo del succión hasta el momento final de la creación del corte llevado a cabo con el láser de femtosegundo.

Los valores de PIO también fueron registrados antes y después del procedimiento mediante tonometría de aplanación «Perkins». Teniendo en cuenta que los niveles elevados de presión presentes durante el experimento pudieran facilitar la vía de evacuación del fluido presente en el ojo, se asumió que si el nivel de presión, después de la cirugía, no era menor de $6 \mathrm{mmHg}$ significaría que no habría fuga de líquido que pudiera invalidar el experimento.

Tabla I. Esta tabla muestra los valores medios, desviación estándar y rango expresados en mmHg para cada ojo, de PIO durante la duración total del experimento: desde la colocación del anillo de succión hasta el final de la creación del flap

\begin{tabular}{lccc}
\hline Ojo & Media & DE & Rango \\
\hline 1 & 101,75 & 18,02 & $59-129$ \\
2 & 76,69 & 35,72 & $34-114$ \\
3 & 99,54 & 33,84 & $36-132$ \\
4 & 109,06 & 35 & $45-160$ \\
5 & 107,72 & 21,54 & $70-141$ \\
6 & 127,35 & 30,1 & $72-157$ \\
7 & 115,35 & 3,18 & $112-122$ \\
\hline \hline
\end{tabular}

\section{RESULTADOS}

Se evaluó la PIO real durante la cirugía en siete ojos de cerdo enucleados.

La media de PIO obtenida durante el procedimiento fue de 84,40 DE 23,83 $\mathrm{mmHg}$ durante el tiempo que duró la succión del ojo y de $122,19 \mathrm{DE}$ 13,58 mmHg durante la creación del flap.

El tiempo medio para completar la succión fue de $40 \mathrm{DE} 9.75 \mathrm{seg}$ (rango, 30-55 seg) y el tiempo medio requerido para crear el flap fue de $52.85 \mathrm{DE}$ $5.66 \mathrm{seg}$ (rango, 50-65 seg) siendo el tiempo medio total de $95.75 \mathrm{DE} 12.39 \mathrm{seg}$.

El nivel medio de PIO de los siete ojos antes de colocar el anillo de succión y comenzar el experimento fue de 11,14 DE 3,43 mmHg llegando a un valor medio al final del procedimiento de 8,85 DE 2,11 mmHg.

\section{DISCUSIÓN}

Hemos diseñado un modelo que permite medir la presión intraocular durante las maniobras quirúrgi-

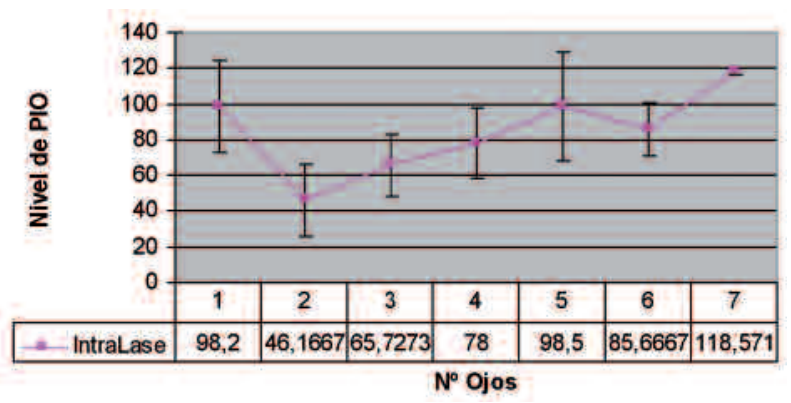

Fig. 1: Esta figura muestra los valores medios y la desviación estándar para cada ojo por separado de PIO, expresada en $\mathrm{mmHg}$, alcanzada durante la fase de succión.

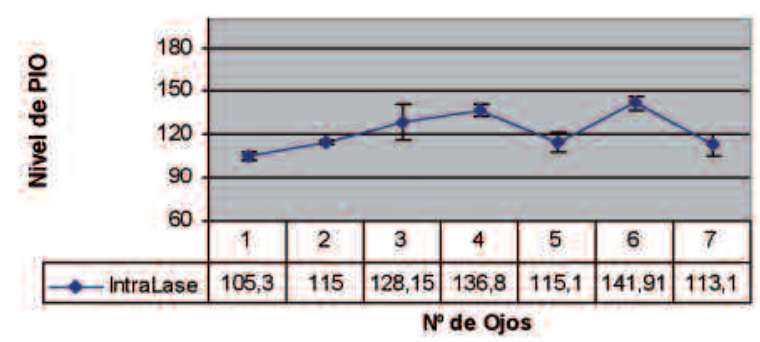

Fig. 2: Esta figura muestra los valores medios y la desviación estándar para cada ojo por separado de PIO, expresada en $\mathrm{mmHg}$, alcanzada durante la fase creación del flap. 
cas realizadas sobre el ojo cuando es sometido a cirugía LASIK.

El uso de la manometría invasiva es una forma habitual de medida de presión intraocular sobre todo en estudios animales. Este método se presenta como la forma más real de registro de la presión empleándose como referencia a la hora de valorar la precisión de otras formas no invasivas de medida.

$\mathrm{Si}$ revisamos la literatura, en unos trabajos la canulación necesaria para el registro con manometría se hace a través de la cámara vítrea mientras que en otros se hace a través de la cámara anterior.

Bajo nuestro criterio, ambas formas de medida pueden mostrar sensibles diferencias entre ellas. Los trabajos de Bissen-Miyajima $\mathrm{H}$ et al. (1) así como los de Kasetsuwan N et al (2) miden la PIO intraocular utilizando un sensor insertado en la cámara vítrea.

De mismo modo, en un experimento llevado a cabo por Flaxel CJ col. (4) en ojos humanos de cadáver se procedió de la misma forma canulando la cámara vítrea para el registro manométrico con el fin de demostrar la relación de la succión empleada durante la cirugía refractiva LASIK y las lesiones retinianas predisponentes en su relación con un cambio en la longitud axial del ojo durante la cirugía.

Sin embargo, otros trabajos realizan la canulación a través de la cámara anterior, como es el caso de los experimentos de Lim JI y col. (5) donde se compara la precisión de la neumotonometría y la tonometría mediante Tono-Pen, en ambos casos tomando como referencia la medida de presión por manometría. Del mismo modo, en otra publicación, Pasaglia CL y col. (6) también emplean el mismo procedimiento de canulación de cámara anterior para la calibración de medidas no invasivas llevadas a cabo con TonPen KL en ojos de gato recién enucleados.

Debemos tener en cuenta que en los experimentos que se llevan a cabo canulando la cámara vitrea, al insuflar una solución glucosada a través del nervio óptico, se consigue cierto grado de licuefacción vítrea lo que facilita el movimiento del fluido. No obstante esta situación hace ciertamente difícil suponer que los valores obtenidos sean reproducibles al depender en gran medida del grado de viscosidad que se ha conseguido durante la preparación del ojo.

Nuestro experimento mantiene mayores similitudes con los trabajos que canulan la cámara anterior que, bajo nuestro punto de vista, hace más fiable el registro de los valores obtenidos.
Otro aspecto importante que aporta validez a método propuesto es el hecho de que la presión intraocular final se mantenga dentro de los valores normales, lo que puede justificar la ausencia de fuga de líquido durante la maniobra, aspecto éste que pudiera haber invalidado el experimento.

Con nuestro modelo de canulación de cámara anterior somos capaces de registras los valores de PIO tanto de la fase de succión (40 DE $9.75 \mathrm{seg}$ ) como de la fase de corte $(52.85 \mathrm{DE} 5.66 \mathrm{seg})$. Si analizamos las fluctuaciones presentes en la PIO, entendida ésta como la desviación estándar presente en cada conjunto de medidas, es interesante resaltar la mayor estabilidad que presenta la fase de corte con respecto a la fase de succión. Este hecho puede ser justificado como consecuencia de la no manipulación del ojo por parte del cirujano en esta fase; y por contra fuente probable de las fluctuaciones presentes durante la fase de succión.

Aún teniendo en cuenta las ventajas que puede aportar este método invasivo de medida de PIO durante determinados procedimiento quirúrgicos, nuestro diseño no está exento de inconvenientes que deben ser tenidos en cuenta para futuras mejoras del mismo.

Por un lado, el hecho de canular la cámara anterior por vía intraescleral puede resultar algo engorroso y puede generar dificultades adicionales al cirujano en el momento de la colocación del anillo de succión.

Además, otra consideración a tener en cuenta es que la medida de PIO que obtenemos puede no ser la que realmente existe en otras partes del ojo, si bien las propiedades físicas de los fluidos nos dicen que la presión ejercida sobre un punto en un compartimento estanco se transmite directamente hacia todos los puntos de ese compartimento. De nuevo, el hecho de que la PIO al final de nuestro experimento se mantuviera dentro de los niveles normales ayuda a pensar que el ojo se comporta como tal compartimento durante todo el tiempo que duró el experimento, transmitiendo por tanto los valores de PIO hacia todos los puntos del interior del ojo.

Recientemente Morris, CA. y col. (7) compararon la precisión de la tonometría no invasiva de rebote con los valores de PIO obtenidos mediante canulación en un experimento animal relacionado con el efecto hipotensor de prostaglandina tópica demostrando que no existían diferencia estadísticamente significativas entre ambas formas de medición. Si tenemos en cuenta que la medida de presión 
por canulación se presenta hasta el momento como la mejor forma de medida real de la PIO, es lógico pensar que si ésta se mide además en la cámara anterior del ojo, evitando los inconvenientes anteriormente mencionados al hacerlo en la cámara vítrea, pudiera ser ésta una forma más acertada de conocer la presión real en el interior del ojo.

\section{BIBLIOGRAFÍA}

1. Bissen-Miyajima H, Suzuki S, Ohashi Y, Minami K. Experimental observation of intraocular pressure changes during microkeratome suctioning in laser in situ keratomileusis. J Cataract Refract Surg 2005; 31: 590-594.

2. Kasetsuwan N, Pangilinan RT, Moreira LL, DiMartino DS, Shah SS, Schallhorn SC, et al. Real time intraocular pressure and lamellar corneal flap thickness in keratomileusis. Cornea 2001; 20: 41-44.

3. Sachs HG, Lohmann CP, Op de Laak JP. Intraocular pressure in sections with 2 microkeratomes in vitro. Ophthalmologe 1997; 94: 707-709.

4. Flaxel CJ, Choi YH, Sheety M, Oeinck SC, Lee JY, McDonnell PJ. Proposed mechanism for retinal tears after LASIK: an experimental model. Ophthalmology 2004; 111: 24-27.

5. Lim JI, Blair NP, Higginbothan EJ, Farber MD, Shaw WE, Garretson BR. Assessment of intraocular pressure in vitrectomized gas-containing eyes. A clinical a manometric comparison of the Tono-Pen to the pneumotonometer. Arch Ophthalmol 1990; 108: 684-688.

6. Passaglia CL, Guo X, Chen J, Troy JB. Tono-Pen XL calibration curves for cats, cows and sheep. Vet Ophthalmol 2004; 7: 261-264.

7. Morris CA, Crowston JG, Lindsey JD, Danias J, Weinreb $R N$. Comparison of invasive and non-invasive tonometry in the mouse. Exp Eye Res 2006; 82: 1094-1099. 